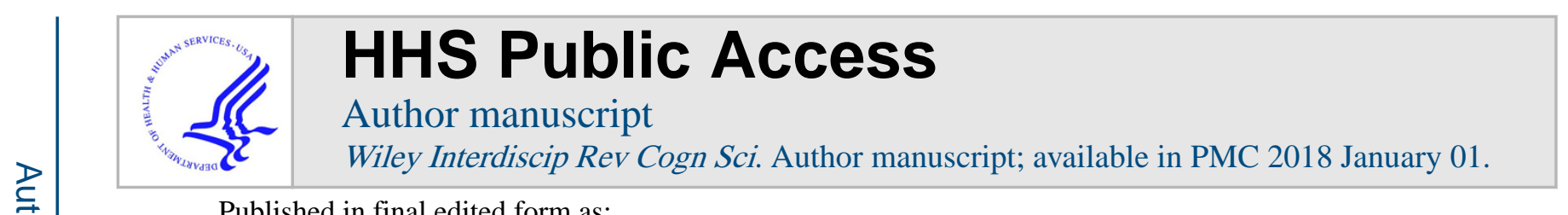

Published in final edited form as:

Wiley Interdiscip Rev Cogn Sci. 2017 January ; 8(1-2): . doi:10.1002/wcs.1402.

\title{
Principles of Brain Development
}

\author{
Joan Stiles \\ Department of Cognitive Science, University of California, San Diego
}

\begin{abstract}
Throughout much of the 20th Century, the major models of brain development were strongly deterministic. It was thought that brain development proceeds via a prescribed blueprint that is somehow innately specified in the organism. Contemporary models present a distinctly different view of both inheritance and brain development. First, we do not inherit blueprints or plans, we inherit genes and the cellular machinery for expressing them. Genes carry essential information for creating proteins, but do not determine biological processes or developmental outcomes; the first cells contain the elements necessary for creating proteins based on the information coded in the nucleotide sequences of genes. Second, brain development is dynamic: the biological state of the brain at any moment is the product of developmental processes that involve an intricate interplay among genes and an ever-expanding range of environmental factors-from local cellular events to influences from the outside world. In science, models matter. They reflect underlying assumptions about how things can happen, and thus influence the kinds of questions we ask, the kinds of experiments we propose, the therapies we develop, and the educational curricula we construct. The dynamic model of brain development accounts for powerful neurobehavioral effects that can simply not be accommodated by deterministic models.
\end{abstract}

\section{Keywords}

Dynamic Models; Predeterminist Models; Interaction; Neural Plasticity; Progressive Differentiation; Progressive Commitment; Temporal Constraints

\section{INTRODUCTION}

The mature human brain contains some eighty-five billion neurons that make trillions of connections with other neurons that underlie all human thought, emotion, action, and behavior ${ }^{1}$. One of the most fundamental questions in modern neuroscience is how this incredibly complex biological system comes into being. In short, how does the human brain develop?

Early models of brain development were strongly deterministic, centered on the idea that development proceeds via a prescribed blueprint that is somehow innately specified in the organism. Contemporary models present a distinctly different view of both inheritance and brain development. Biological inheritance involve two important factors: The first is DNA, which is made up of the nucleotide sequences (the genes) that serve as a template for producing proteins. Proteins, not genes, are the active agents in all developmental processes. However, DNA has never been inherited in isolation. DNA inheritance always involves the second inherited factor, the first cell, which contains both the DNA and the cellular 
machinery required to transcribe the information in the DNA into proteins. By itself, DNA is inert. It is only though the transforming processes of DNA transcription and RNA translation that the information in the DNA is rendered active. Thus, at conception, the child inherits both the DNA and the first environment (the cell) that together provide the tools necessary for all subsequent development, including brain development ${ }^{2-4}$. Neither genes nor environment factors prescribe outcomes. The biological state of the organism at any moment is the product of developmental processes that involve an intricate interplay among complex cascades of gene expression interacting with influences from an ever-expanding range of environmental factors - from local cellular events to influences from the outside world ${ }^{5}$.

Brain development proceeds over a protracted period of time and involves the complex interaction of molecular (genetic), cellular, and environmental systems and elements ${ }^{6,7}$. It is also progressive: Structures and systems that emerge at one point in development often set the stage for the development of subsequent systems. This essay will review three key concepts of this dynamic process: neural plasticity, progressive differentiation and commitment, and sources of developmental constraint. Each of these topics draws upon examples that illustrate brain development as progressive, dynamic, and adaptive, rather than innately specified.

\section{NEURAL PLASTICITY}

Fundamentally, brains are adaptive. They adjust to various contingencies, be they biological or environmental. The term neural plasticity refers to this broad adaptability on virtually all levels of the neurocognitive system, from molecules to behavior ${ }^{7,8}$. Plasticity operates throughout the course of typical brain development. It is seen, for example, in the neural networks for face processing (see Haist, $X X X$, WIREs $X X X$, also in the collection How We Develop). There we observe that children activate a more extensive set of brain structures compared to adults. As face processing expertise is acquired, neural networks are pruned back such that only the most efficient and effective components of the face network are engaged.

The effects of neural plasticity are perhaps most evident in conditions of pathology (see D'Sousa and Karmiloff-Smith, Neurodevelopmental disorders, WIREs Cog Sci, also in the collection How We Develop). A wide range of neuropathological events can impact the trajectories of development and thereby alter basic patterns of brain organization. These alterations reflect the underlying neuroplasticity of the immature brain as the developing system adapts to the effects of neural insult. Although plasticity is most evident in the young organism, it is also observed on a more limited scale in the adult brain (see Power and Schlaggar, Neural plasticity across the lifespan, WIREs Cog Sci, also in the collection How We Develop). Many studies have demonstrated that the mature brain can reorganize itself (at least within local brain areas) in response to injury 9,10 . Thus, at every point in development and at every level of the neural system, the capacity to adapt is essential to the functional viability of the organism. Indeed, failure to adapt can have catastrophic consequences. As such, neural plasticity is a central feature of brain development ${ }^{11}$. 


\section{PROGRESSIVE DIFFERENTIATION AND PROGRESSIVE COMMITMENT}

Progressive differentiation and progressive commitment represent two fundamental and complementary aspects of brain development ${ }^{12}$. Progressive differentiation refers to the gradual elaboration of the neural system, including differentiation of distinct cell types, patterns of connections, and different functions within neural networks (see Darnell and Gilbert, Neuroembryology, WIREs Dev Biol, also in the collection How We Develop). Early in development, the embryo begins as a relatively undifferentiated bundle of cells. During the third week after conception, molecular signaling between subgroups of cells induces a change in one group of cells that results in their differentiation to neural stem cells. The neural stem cells are also called neural progenitor cells because they are the cells that will later give rise to all other neurons and many support cells (see Jernigan, Construction of the human forebrain, WIREs $X X X$, also in the collection How We Develop). The differentiation of neural stem cells also has a spatial component critical for establishing the basic functional organization of the embryo. Concurrent with signaling that promotes differentiation, other signaling induces neural stem cells in rostral regions to become forebrain progenitors and more caudally positioned cells to become spinal and hindbrain progenitors-subgroups of neural stems cells that will later give rise to distinct populations of neuron in the forebrain, hindbrain, and spinal cord. The complexity of progressive differentiation suggests a very different model of brain development than that offered by more deterministic models.

Progressive commitment is closely related and complementary to progressive differentiation, reflecting processes associated with the stabilization of neural elements and systems. As discussed earlier, developing systems exhibit considerable plasticity and capacity to adapt to varying signals and contingencies. However, that plasticity declines with development as different neurocognitive elements become progressively committed to particular systems ${ }^{13}$. This phenomenon of progressive commitment is illustrated well by "monocular deprivation" studies in which an eye is deprived of light stimulation early in development (see Power and Schlaggar, Neural plasticity across the lifespan, WIREs Cog Sci, also in the collection How We Develop). Those studies were designed to examine the effect of early deprivation on a critical feature of primary visual cortex organization, the ocular dominance column (ODC). ODCs occur because the neural inputs from each eye are clustered within visual cortex, creating striking banding patterns. In the monocular deprivation studies, one eyelid of young monkeys was sutured shut. In the youngest monkeys, this period of deprivation induced reorganization in the ODCs. Specifically, the bands associated with the deprived eye shrank significantly, while the bands associated with the active eye expanded into the territory of the deprived eye. These findings clearly demonstrated input-driven plasticity in the developing brain. Critically, the magnitude of the effect waned with increasing delays in the onset of deprivation. Specifically, the greatest effects of deprivation were observed in the youngest monkeys (approximately 6 weeks old); by one year of age, no effects of deprivation could be detected. This illustrates the principle of progressive commitment.

Progressive differentiation and commitment represent complementary aspects of brain development. They reflect the essential balance between the capacity for adaptation and the need for stability in functional systems. The two constructs convey the temporal nature of 
brain development. At all levels of the neural system, differentiation coupled with commitment appears to be the governing principle of brain development.

\section{CONSTRAINTS ON BRAIN DEVELOPMENT}

From the very beginning, brain development is influenced by both intrinsic factors ranging from molecular cues derived from gene expression to cell-cell interaction to system activation, and by extrinsic factors arising from input from sources outside the organism. Neither set of factors acts in isolation to determine developmental outcome. Rather, they work in concert as part of a complex and dynamic system that serves to support and guide the development of the brain. This is a model of neural development that is anchored in the process of development itself, with each step influenced by myriad cues arising from multiple levels of the emerging system. Indeed, one might legitimately ask whether such a model is too dynamic. There are many degrees of freedom in these complex, interactive signaling cascades, but if there is no specific mechanism for determining a particular outcome, then how it is that development proceeds with such uniformity to produce species typical organisms? The answer to this question lies in the fact that while development is dynamic it is occurs in the context of three powerful constraints that originate from three principal sources: genetics, environment, and time.

Genes are vital molecular tools. They provide resources for creating particular proteins essential to many critical aspects of development. Each species (and each individual) has a specific set of genes that has been acquired across the course of evolution. While genes do not prescribe developmental processes, their availability at the right time and in the right quantity provides powerful constraints on how development can proceed. For example, in the early patterning of sensory and motor cortical areas, complementary concentration gradients of different molecular signals establish the initial organization (see Figure 5 in Power and Schlaggar, Neural plasticity across the lifespan, WIREs Cog Sci, also in the collection How We Develop). For example, Pax6 is expressed in a gradient that is most concentrated in anterior regions of the developing neural plate and becomes gradually less concentrated in more and more posterior regions. Emx2 shows the opposite pattern of expression. Specification of motor, somatosensory and visual regions in the neural plate depends on the exact combination of these two signaling molecules in particular concentrations (i.e. high Pax6 + low Emx2 gives rise to motor areas, while high Emx2 + low Pax6 gives rise to visual areas). In experimental studies in rats the level of expression of one two gene products was altered to weaken its expression gradient, thus altering the precise combination of signals to cells in particular regions of the neural plate. As a result the patterning of cortical was changed. Thus it is the combination of multiple molecular signals present in varying concentrations in different areas that induces the differentiation of functionally distinct regions within the developing neocortex. Critically, when the concentration of one protein is experimentally increased or decreased, the cortical maps shift, reflecting altered patterns of molecular signaling ${ }^{14}$.

Like genes, the environment imposes rigorous constraints on how an organism can develop. From an evolutionary perspective, development is an adaptation to the contingencies of the environment. Early development relies on what Greenough has called experience-expectant 
change ${ }^{15-17}$. Normal development requires normal input from the world to modulate and shape the emerging functional organization of neural systems. Neural systems do not develop normally in the absence of typical environmental input. The monocular deprivation studies discussed above provide powerful examples of the importance of normal, expected input on developing systems. The environment also plays just as powerful a role in the development of neurobehavioral systems. For example, studies in severely deprived human infants illustrate the wide-ranging effects of developmental conditions on many aspects of neural, emotional, and cognitive health For example overall brain size is smaller in severely deprived children. More specifically, regions of the amygdala and prefrontal cortex as well as the pathways that connect them are also abnormal. This is an important neural circuit mediating emotion, and the observed neural abnormalities likely underpin the difficulties with emotional regulation often observed in these children. Similar abnormalities are observed in neural systems that mediate language, visuospatial processing and attention (see Bick and Nelson, Early experience and brain development, WIREs Cog Sci, also in the collection How We Develop).

A third essential constraint arises from time. The integrity of the developmental process depends on the availability of the right neural elements appearing at the appropriate moment. For example, the differentiation of neural stem cells during early embryonic development sets the stage for the formation of the ventricular zone, a region deep in the brain that contains the neural progenitor cells. The formation of the ventricular zone then sets the stage for the production and scaffolding of neurons to form the neocortex. Failure at any of these crucial steps will lead to a catastrophic failure of brain development. This process is not dictated by genes, but emerges through time. Thus, brain development is temporally constrained; the developing organism creates tools on the fly necessary for each successive step in development. At each moment, the organism has both a state and a history that limit both what can affect it immediately and its future development. For example, whereas light stimulation contributes little to development in fetuses, it is essential for the typical development of the visual system in newborns. Time limits what changes can occur and what factors can influence development. In that sense, development is a self-organizing process.

The trajectory of individual neurocognitive development emerges over time through ongoing interactions across multiple levels of the neurobehavioral system (see Brown, Individual differences in human brain development, WIREs Cog Sci, also in the collection How We Develop). The processes of progressive differentiation and commitment continuously modify the existing state of the organism such that the system is reorganized not once, but many times over the course of development. In that sense, development can be construed as a process of continuous, successive reorganization. The product of these developmental processes is a relatively stable (though still plastic) organization in the adult brain.

\section{SUMMARY}

Brain development is dynamic and adaptive. It is temporally defined and constrained by both genetic and experiential factors. This dynamic model differs significantly from older maturational models in which systems emerge in a linear, predetermined fashion. It is

Wiley Interdiscip Rev Cogn Sci. Author manuscript; available in PMC 2018 January 01. 
supported by a growing body of data on brain development and by studies examining associations between neural and behavioral development.

Our scientific models reflect underlying assumptions about how things can happen, and thus influence the kinds of we questions we ask, the kinds of experiments we propose, the therapies we develop, and the educational curricula we construct. The dynamic model of brain development accounts for powerful neurobehavioral effects that can simply not be accommodated by deterministic models. Recent investigations of neurodevelopmental disorders, early deprivation, and individual variation not only support a dynamic model of neurobehavioral development, they also reframe how we think about the brain.

\section{Acknowledgments}

This work was supported by the National Institute of Child Health and Human Development Grant R01-HD25077, National Institute of Neurological Disorders and Stroke Grant \#P50-NS22343, and National Institute of Deafness and Communicative Disorders Grant \# P50-DC01289.

\section{References}

1. Azevedo FA, Carvalho LR, Grinberg LT, Farfel JM, Ferretti RE, Leite RE, Jacob Filho W, Lent R, Herculano-Houzel S. Equal numbers of neuronal and nonneuronal cells make the human brain an isometrically scaled-up primate brain. J Comp Neurol. 2009; 513:532-541. [PubMed: 19226510]

2. Jablonka E. Information: Its interpretation, its inheritance, and its sharing. Philosophy of Science. 2002; 69:578-605.

3. Keller, E. The century of the gene. Cambridge, MA: Harvard University Press; 2000.

4. Keller, E. Decoding the genetic program: or, some circular logic in the logic of circularity. In: Beurton, P.; Falk, R.; Rheinberger, H-J., editors. The concept of the gene in development and evolution: Historical and epistemological perspectives. Cambridge: Cambridge University Press; 2000. p. 159-177.

5. Sarkar S. Information in genetics and developmental biology: Comments on Maynard Smith. Philosophy of Science. 2000; 67:208-213.

6. Gottlieb G. Conceptions of prenatal development: behavioral embryology. Psychol Rev. 1976; 83:215-234. [PubMed: 188059]

7. Stiles, J. The Fundamentals of Brain Development: Integrating Nature and Nurture. Cambridge, MA: Harvard University Press; 2008.

8. Berlucchi G, Buchtel HA. Neuronal plasticity: historical roots and evolution of meaning. Exp Brain Res. 2009; 192:307-319. [PubMed: 19002678]

9. Hubener M, Bonhoeffer T. Neuronal plasticity: beyond the critical period. Cell. 2014; 159:727-737. [PubMed: 25417151]

10. Kolb B, Gibb R. Plasticity in the prefrontal cortex of adult rats. Front Cell Neurosci. 2015; 9:15. [PubMed: 25691857]

11. Kalia M. Brain development: anatomy, connectivity, adaptive plasticity, and toxicity. Metabolism. 2008; 57(Suppl 2):S2-5. [PubMed: 18803960]

12. Stiles, J.; Brown, T.; Haist, F.; Jernigan, T. Brain and Cognitive Development. In: Lerner, R.; Liben, L.; Meuller, U., editors. Handbook fo Child Psychology and Developmental Neuroscience. New York: Wiley; 2015. p. 9-62.

13. Pascual-Leone A, Amedi A, Fregni F, Merabet LB. The plastic human brain cortex. Annu Rev Neurosci. 2005; 28:377-401. [PubMed: 16022601]

14. Tropea D, Van Wart A, Sur M. Molecular mechanisms of experience-dependent plasticity in visual cortex. Philos Trans R Soc Lond B Biol Sci. 2009; 364:341-355. [PubMed: 18977729] 
15. Black, J.; Greenough, W. Induction of pattern in neural structure by experience: Implications for cognitive development. In: Lamb, M.; Brown, A.; Rogoff, B., editors. Advances in developmental psychology. Hillsdale, NJ: Erlbaum; 1986. p. 1-50.

16. Greenough, W. Experience and brain development: An update in Brain development and cognition: A reader. Oxford: Blackwell; 1993.

17. Greenough WT, Black JE, Wallace CS. Experience and brain development. Child Dev. 1987; 58:539-559. [PubMed: 3038480]

\section{FURTHER READING}

18. Stiles, J.; Brown, TT.; Haist, F.; Jernigan, T. Brain and Cognitive Development. In: Lerner, RM.; Liben, LS.; Meuller, U., editors. Handbook of Child Psychology and Developmental Neuroscience. New York: Wiley; 2015. p. 9-62.

19. Gilbert SF. All I Really Needed to Know I Learned during Gastrulation. CBE-Life Sciences Education. 2008; 7(112-13) http://www.lifescied.org.

20. Gottlieb G. Conceptions of prenatal development: behavioral embryology. Psychol Rev. 1976; 83:215-34. [PubMed: 188059] 